\title{
Measurement of the optical activity of anisotropic samples by transmission Mueller matrix ellipsometry
}

\author{
Oriol Arteaga ${ }^{1, a}$ and Adolf Canillas ${ }^{1}$ \\ ${ }^{1}$ FEMAN Group, Departament de Física Aplicada i Òptica, IN²UB, Universitat de Barcelona, Barcelona 08028, Spain
}

\begin{abstract}
In this work we review some of the approaches we have developed to study the optical activity in anisotropic samples. They all are based in the spectroscopic measurement of the normalized Mueller matrices of the samples.
\end{abstract}

\section{Introduction}

Measurement of molecular optical activity in the visible and ultraviolet is performed as a routine task in chemistry and biology laboratories. Standard measurements are performed on commercial instruments: the most usual ones are called dichrographs or CD spectropolarimeters and focus on the detection of the circular dichroism (CD). Nevertheless some models also permit the measurement of the circular birefringence (CB) by the attachment of an accessory. These instrument are usually designed to spectroscopically measure one single element of the Mueller matrix.

The transmission Mueller matrix for a chiral sample that does not exhibit linear birefringence or linear dichroism (this type of sample will be referred in this work as isotropic) is particularly simple:

$$
\mathbf{M}_{\text {iso }}=\left(\begin{array}{cccc}
m_{00} & 0 & 0 & \mathrm{CD} \\
0 & m_{11} & \mathrm{CB} & 0 \\
0 & -\mathrm{CB} & m_{22} & 0 \\
\mathrm{CD} & 0 & 0 & m_{33}
\end{array}\right),
$$

where $m_{00}, m_{11}, m_{22}, m_{33}$ are in most cases close to $1^{1}$. For these type of samples a measurement of the Mueller matrix elements $m_{03}$ and $m_{12}$ (or $m_{30}$ and $-m_{21}$ ) give, respectively, a direct measurement of CD and CB. However, it is well-known that if the studied medium presents linear birefringence or linear dichroism, the standard approaches to measure optical activity may fail because none of the Mueller matrix elements is a direct measurement of CD or $\mathrm{CB}$. In this condition the measurement of single elements of the Mueller matrix gives complicate mixtures of different optical effects rather than pure values of $\mathrm{CD}$ or $\mathrm{CB}$ [1].

\footnotetext{
a e-mail: oarteaga@ub.edu

1 This is true for media without large CD or CB, i. e. in almost all samples where the optical activity has a molecular origin. However, in crystals, for example, the optical activity arises due to helical structural arrangement and they can present large CB. Therefore, the diagonal elements of their Mueller matrices may not be close to 1 .
}

In this work we will present some of the approaches we have developed to study optical activity in anisotropic samples. Our method is based on measuring the complete normalized Mueller matrix of the sample by using a spectroscopic polarimeter and then using an algebraic analysis of the measured Mueller matrices to retrieve CD and CB. We will show that this is a versatile method which can be applied to several different types of samples. The procedure will be exemplified by showing the results obtained for a stirred solution, in which the optical activity is due to the chirality of the molecular constituents, and also for quartz crystal, in which the optical activity is not from a molecular origin but from a chiral structural arrangement.

\section{Instrumental}

The instrument used to spectroscopically measure the Mueller matrices is a two-modulator generalized ellipsometer (2MGE) [2,3]. Such instrument uses two polarizer-photoelastic modulator (PEM) pairs $^{2}$, one as a polarization state generator (PSG) and the other as polarization state analyzer (PSA). On a single configuration this instrument measures 8 independent parameters that correspond to 8 different elements of the Mueller matrix. Complete normalized Mueller matrices are measurable by changing the azimuthal orientations of the PSG and PSA.

Fig. 1 displays the basic optical elements that compose the 2-MGE. As presently configured the 2-MGE described here is prepared to work in the spectral range from 210 to $250 \mathrm{~nm}$. To detect molecular optical activity the instrument needs high sensitivity, since typical $C B$ and $C D$ values in molecular optical active samples are around $0.01 \mathrm{rad}$ or lower. This is achieved by using a software-based Fourierlike synchronous detection system in which the oscillation frequencies of the PEMs as well as some of their multiples or combinations are used as reference signals. The calibration of the instrument is well documented [3] and the

\footnotetext{
${ }^{2}$ Most of modern CD spectropolarimeters use only a polarizerPEM pair that is placed in front of the sample
}

This is an Open Access article distributed under the terms of the Creative Commons Attribution-Noncommercial License 3.0, which permits unrestricted use, distribution, and reproduction in any noncommercial medium, provided the original work is properly cited. 


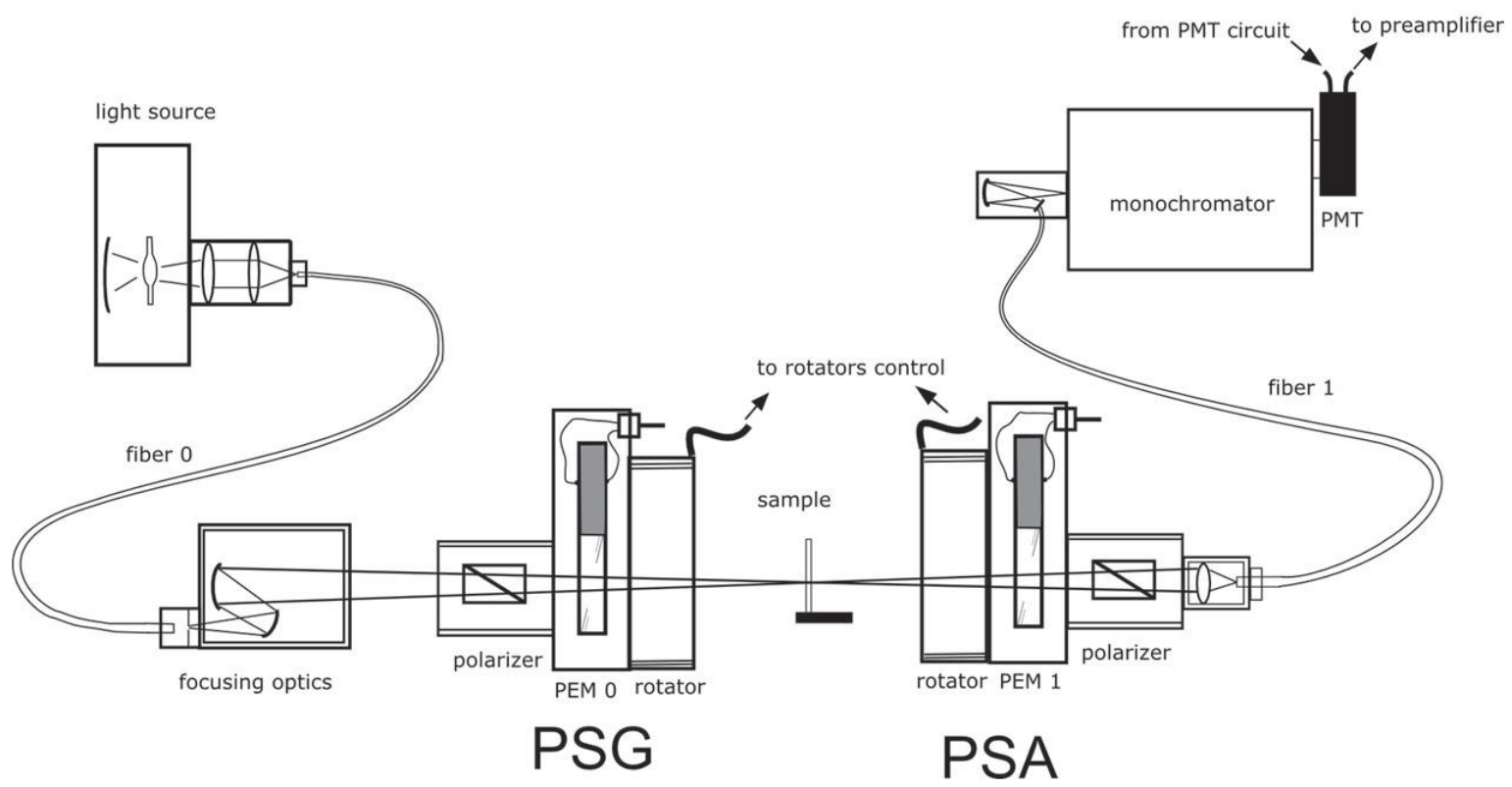

Fig. 1. Scheme of the optical setup used in the transmission 2-MGE.

calibration parameters of the photoelastic modulators are determined easier than in instruments with a single PEM.

The 2-MGE can be operated in reflection and transmission. In reflection the 2-MGE can be used, for example, to characterize thin films ${ }^{3}$, as it is one of the typical applications of ellipsometry; while in transmission [4] (the configuration displayed in Fig. 1) it acts as Mueller polarimeter and, among other applications, it can be used to study optical active media.

\section{Analysis of experimental Mueller matrices. How to obtain $C D$ and $C B$ ?}

There is not a single way to analyze the experimentally measured Mueller matrices. Obviously the analysis may depend on the type of sample under study. In this work we will consider that we are studying light transmitted through samples that are homogeneous in the direction of propagation of light, which basically means that the properties of the sample do not change in depth. Light propagation in anisotropic media under such conditions was first studied by Jones in 1948 [5]. He developed an algebraic approach based on infinitesimal matrices, the $\mathrm{N}$-matrices, which are the decomposition of Jones matrix into a large number of identical matrix components infinitesimally close to the identity matrix.

By using the results of Jones work it is possible to find simple analytic equations [6] that allow to invert any Jones matrix or any nondepolarizing Mueller matrix into the basic polarization properties: $\mathrm{CD}, \mathrm{CB}$ and the linear birefringence and linear dichroism. However, as it is described in

\footnotetext{
${ }^{3}$ Including anisotropic samples because it is a generalized ellipsometer.
}

[6], for experimental Mueller matrices this method is not directly applicable because experimental Mueller matrices usually contain some level of depolarization. Thus, to be able to use those equations it is necessary to calculate first a non-depolarizing estimate of the experimental Mueller matrix. Depending on how close is the experimental matrix to a non-depolarizing Mueller matrix the application of this method will result in more or less reliable results.

Another possibility to retrieve $\mathrm{CD}$ and $\mathrm{CB}$ from experimental Mueller matrices consist in the application of an algorithm to decompose the Mueller matrices. A matrix decomposition expresses a matrix as a function of other matrices which indicate useful properties. The Lu-Chipman decomposition [7], for example, expresses a Mueller matrix as the matrix product of pure diattenuator, pure retarder and depolarizer matrices. In this case CD would be obtained from the diattenuator matrix and $\mathrm{CB}$ from the retarder. In this decomposition the three components can occur in several different orders [8], and the values of each component (and thus the values of $\mathrm{CD}$ and $\mathrm{CB}$ ) may change based on the order, which is an unsatisfactory result if one expects a univocal result.

To sort this difficulty we have worked in a similar decomposition, that we named as pseudopolar decomposition [9], that is order independent for the diattenuator and retarder factors. This can be done by adding several correction factors that account for the non-commuting optical effects of the sample (i. e. the effects that depend on the order in which the matrices factor are multiplied). This decomposition ensures that, respectively, the birefringent effects included in the retarder factor and the dichroic effects of the diattenuator factor correspond with the birefringent and dichroic terms of the experimental matrix.

Some Mueller matrices corresponding to depolarizing media are better analyzed with a symmetric decomposi- 


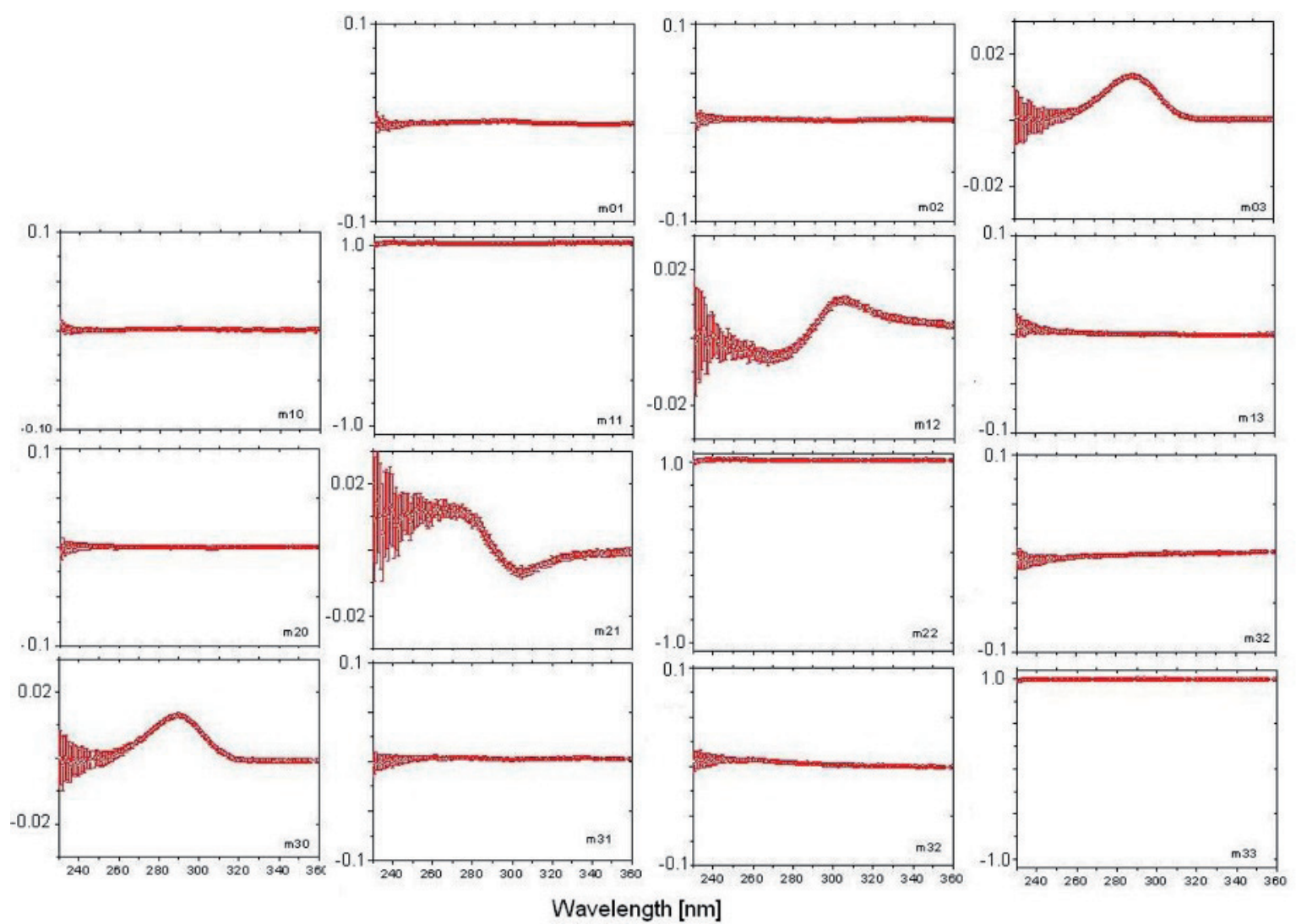

Fig. 2. Normalized spectroscopic Mueller matrix of an isotropic chiral solution contained in a $1 \mathrm{~mm}$ pathlength cuvette.

tion [10] in which the depolarization factor is placed "in the middle" of an optical sequence containing diattenuators and retarders. Unlike the decompositions cited before, the depolarization factor of the symmetric decomposition is a simple diagonal Mueller matrix that characterizes better the depolarization behaviour introduced by a number of depolarizing media.

\section{Examples}

\subsection{Isotropic optical active solution}

This first example we show does not correspond to an anisotropic sample. In this example we just measured a chiral solution of camphorsulphonic acid which is usually used as a standard of calibration for commercial CD instruments.

Fig. 2 shows the spectroscopic Mueller matrix of a camphorsulphonic acid solution which contains a clear band of CD absorption (and its correspondent dispersive CB) at around $290 \mathrm{~nm}$. It can be clearly seen that his measurement reproduces the Mueller matrix pattern given in Eq. [1].

\subsection{Stirring supramolecular solution of fibre-like aggregates}

In this experiment a solution containing fibre-like aggregates of porphyrin $\mathrm{TPPS}_{4}$ was stirred with a small magnetic stirred in clockwise $(\mathrm{CW})$ and anti-clockwise(ACW) directions. This system is anisotropic because the long shaped aggregates get preferential orientations. Measurements of the Mueller matrix are taken in situ while stirring and show the reversible induction of optical activity in the solution. The aggregates fold and twist by effect of the hydrodynamic shear gradients created by the stirring vortex [11, 12].

Panel a of Fig. 3 shows the spectroscopic Mueller matrices for the aggregated solution measured in the three configurations: stagnant, $\mathrm{CW}$ stirring and $\mathrm{ACW}$ stirring. Panel $\mathrm{b}$ shows the physical parameters calculated from the experimental Mueller matrices by the pseudopolar decomposition algorithm. An induction of the optical activity in the solution is observed. It is determined by the stirring direction and it is a completely reversible phenomenon.

\section{$4.3 \alpha-\mathrm{SiO}_{2}$ crystal}

Quartz is a very common noncentrosymmetric quartz enantiomorphous crystal. It has been extensively studied from the beginning of the XIXth century, actually the firsts studies about optical activity by Arago, Biot and Fresnel were done on quartz. Most of measurements on the optical activity of quartz have in common they have been performed in the unique direction of the crystal in which the crystal does not show linear birefringence. This corresponds to light propagating parallel to the optical axis. However, it is wellknown from crystallographic fundamentals that quartz also 


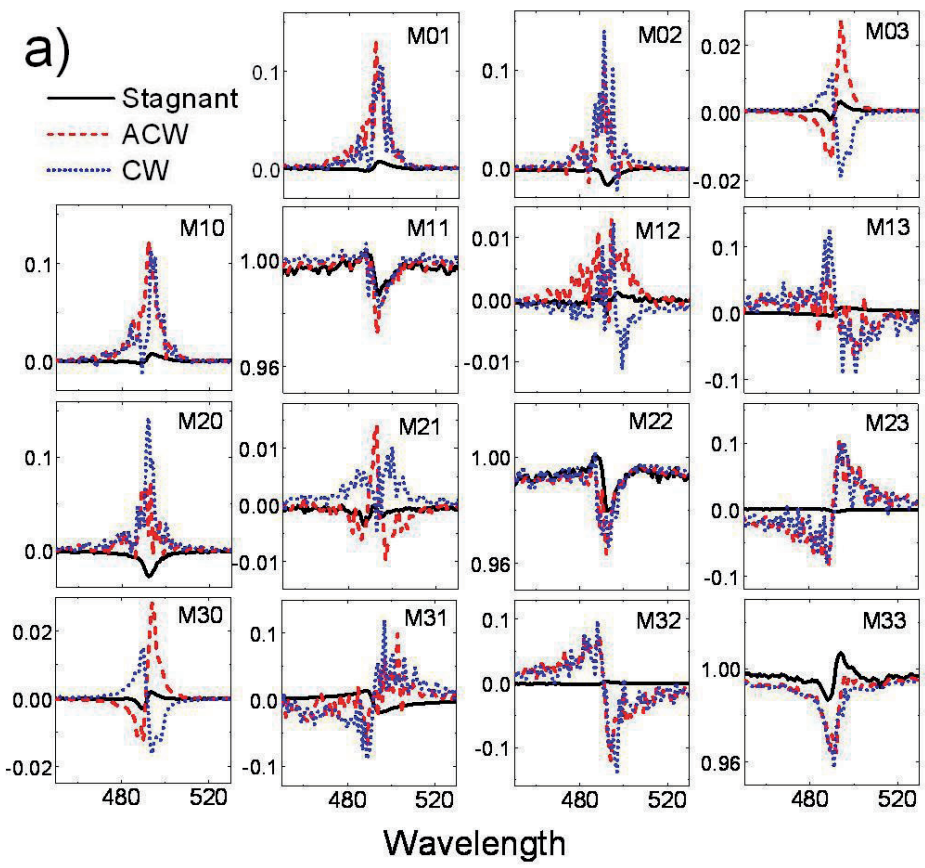

b)
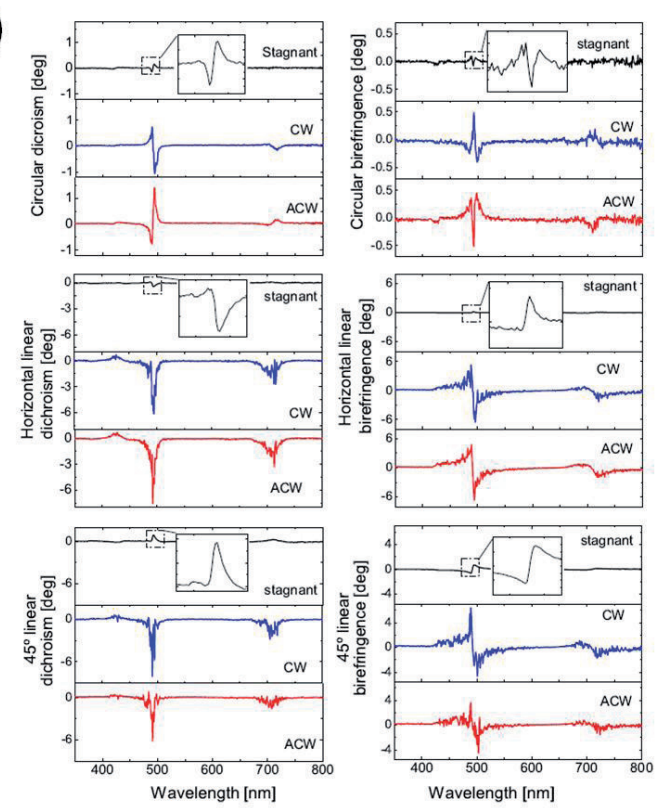

Fig. 3. a), Mueller Matrix of the stirred porphyrin solution. b), Parameters found after the application of the pseudopolar decomposition on matrices of panel a.

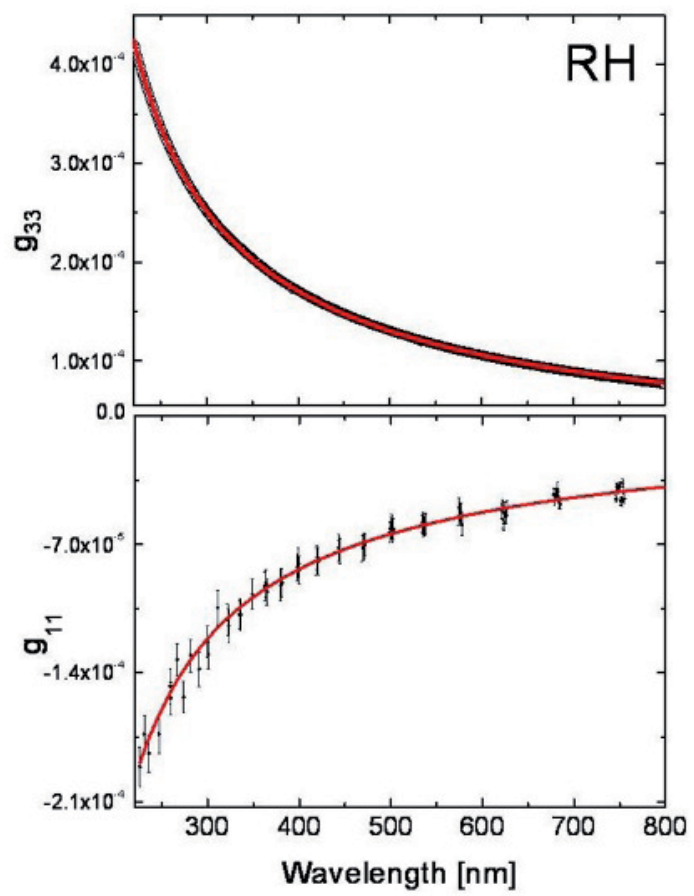

Fig. 4. Spectroscopic determination of the components of the gyration tensor of quartz for a right-handed quartz crystal plate.

exhibits optical activity (in this case only $\mathrm{CB}$ and not $\mathrm{CD}$, because quartz does not absorb at uv-vis range) for directions other than the optic axis, but for these directions the linear birefringence is higher than the CB and "hides" its effect. In these circumstances, the measurement of $\mathrm{CB}$ becomes extremely complex. The $\mathrm{CB}$ dependence on the di- rection of light propagation in quartz can be rigorously expressed in terms of its gyration tensor, which has two different components: $g_{33}$ for directions parallel to the optic axis, which is easy to measure, and $g_{11}$, for directions perpendicular to the optic axis, of difficult measurement.

Our approach to measure the $g_{11}$ gyration component is based on the measurement of the transmission Mueller matrix at oblique incidence of a quartz plate that is cut such that the optical axis lies perpendicular to the crystal surface. Considering the geometry of this experiment we can spectroscopically calculate the values of $g_{11}$ from the measured Mueller matrix if the following parameters are also known or measured with anticipation: $g_{33}$, angle of incidence and extraordinary and ordinary refractive indexes. The details of the experiment are given in [13].

Fig. 4 shows the measured gyration tensor components for a plate made of right-handed quartz. For left-handed quartz the gyration tensor components have the opposite signs with respect to the right-handed case.

\section{Discussion and conclusion}

The measurement of the complete Mueller matrix yields more information than traditional CD or CB spectroscopy techniques and, for chiral anisotropic samples, ensures measurements free from contributions of other optical effects (linear dichroism and linear birefringence). From an experimental point of view the transmission 2-MGE is an ideal instrument for this purpose because it allows the measurement of the complete Mueller matrix and retains the high sensitivity and wide spectral range characteristics of phase modulated ellipsometry. The experimentally deter- 
mined Mueller matrices must be analyzed to unveil the basic polarization effects ( $\mathrm{CB}, \mathrm{CD}$ and others) of the samples. Probably there is not a universal method to perform this analysis because it depends on the type of sample: for homogeneous media that do not depolarize there exist analytic equations to retrieve these effects, whereas for depolarizing media Mueller matrix decomposition techniques can be used. The presented examples concern drastically different types of samples, but we have shown that, essentially, fairly similar approaches can be used to study these systems. The spectroscopic measurement of the normalized Mueller matrices of the samples is, to our knowledge, the most adequate measurement technique to study optical activity in anisotropic media.

\section{References}

1. Freudenthal, J. H., Hollis, E., and Kahr, B., "Imaging chiroptical artifacts", Chirality 21(1E), E20-E27 (2009).

2. Jellison, G. E. and Modine, F. A. Appl. Opt. 36(31), 8190-8198 (1997).

3. Jellison, G. E. and Modine, F. A. Appl. Opt. 36(31), 8184-8189 (1997).

4. Jellison, G. E., Griffiths, C. O., Holcomb, D. E., and Rouleau, C. M. Applied optics 41(31), 6555-6566 (2002).

5. Jones, C. R. J. Opt. Soc. Am. 38(8), 671-685 (1948).

6. Arteaga, O. and Canillas, A., "Analytic inversion of the Mueller-Jones polarization matrices for homogeneous media", to appear in Opt. Lett., published online (2010).

7. Lu, S.-Y. and Chipman, R. A. J. Opt. Soc. Am. A 13(5), 1106-1113 (1996).

8. Ossikovski, R., De Martino, A., and Guyot, S. Opt. Lett. 32(6), 689-691 (2007).

9. Arteaga, O. and Canillas, A. J. Opt. Soc. Am. A 26(4), 783-793 (2009).

10. Ossikovski, R. J. Opt. Soc. Am. A 26(5), 1109-1118 (2009).

11. Arteaga, O., Escudero, C., Oncins, G., El-Hachemi, Z., Llorens, J., Crusats, J., Canillas, A., and Ribó, J. M. Chemistry - An Asian Journal 4(11), 1687-1696 (2009).

12. Arteaga, O., Canillas, A., Purrello, R., and Ribó, J. M. Opt. Lett. 34(14), 2177-2179 (2009).

13. Arteaga, O., Canillas, A., and Jellison, J. Appl. Opt. 48(28), 5307-5317 (2009). 